\title{
Spectral Properties of Image Measures Under the Infinite Conflict Interaction
}

\author{
Sergio Albeverio, Volodymyr Koshmanenko, Mykola Pratsiovytyi \\ and Grygoriy Torbin
}

\begin{abstract}
We introduce the conflict interaction with two positions between a couple of image probability measures and consider the associated dynamical system. We prove the existence of invariant limiting measures and find the criteria for these measures to be a pure point, absolutely continuous, or singular cotinuous as well as to have any topological type and arbitary Hausdorff dimension.
\end{abstract}

Mathematics Subject Classification (2000). 11A67; 11K55; 26A46; 28A80; 28D05; $60 \mathrm{G} 30$.

Keywords. Conflict interaction, Hausdorff dimension, image measure, probability measure, singular measure, stochastic matrix.

\section{Introduction}

Let $(\Omega, \mathcal{F})$ be measurable space, let $\mathbb{P}$ be a class of probability measures on $\mathcal{F}$, and let ${ }^{*}$ be a noncommutative binary algebraic operation defined for elements of $\mathbb{P}$. A measure $\mu \in \mathbb{P}$ can be interpreted as a measure of 'influence' on 'controversial territory' for some 'subject of controversy'.

If two non-identical measures $\mu$ and $\nu$ are not mutually singular, then they are called conflict measures and an operation $*$ represents the mathematical form of the conflict interaction between $\mu$ and $\nu$.

Given $\mu, \nu \in \mathbb{P}$ let us consider a sequence of paris $\mu^{(n)}, \nu^{(n)} \in \mathbb{P}$ of measures defined as follows:

$$
\begin{array}{ll}
\mu^{(1)}=\mu * \nu, & \nu^{(1)}=\nu * \mu ; \\
\mu^{(2)}=\mu^{(1)} * \nu^{(1)}, & \nu^{(2)}=\nu^{(1)} * \mu^{(1)} ; \ldots \\
\mu^{(n+1)}=\mu^{(n)} * \nu^{(n)}, & \nu^{(n+1)}=\nu^{(n)} * \mu^{(n)} ; \ldots
\end{array}
$$

By this each operation $*$ defines an mapping $g(*): \mathbb{P} \times \mathbb{P} \rightarrow \mathbb{P} \times \mathbb{P}$ and generates a certain dynamical system $(\mathbb{P} \times \mathbb{P}, g(*))$. The following problems are of interest: 
(1) existence of invariant points and invariant sets of $(\mathbb{P} \times \mathbb{P}, g(*))$;

(2) descriptions of the limiting measures

$$
\mu^{\infty}=\lim _{n \rightarrow \infty} \mu^{(n)} ; \nu^{\infty}=\lim _{n \rightarrow \infty} \nu^{(n)} ;
$$

(3) topological, metric, and fractal properties of the limiting measures and dependence of these properties on the conflict interaction.

In $[6,7]$ a variant of a conflict interaction $*$ for discrete measures on finite and countable spaces was discussed. In this paper we involve in consideration the cases of continuous measures. More precisely, here we handle with measures $\mu, \nu$ which are image measures of infinite products of discrete measures. We prove the existence of the limiting invariant measures $\mu^{\infty}, \nu^{\infty}$ and show that they are mutually singular if $\mu \neq \nu$. We find necessary and sufficient conditions for these measures to be pure absolutely continuous, pure singular continuous or pure discrete resp. Metric, topological and fractal properties of the supports of the limiting measures are studied in details. We show that by using rather simple construction one can get a singular continuous measure $\mu^{\infty}$ with desirable fractal properties of its support, in particular, with any Hausdorff dimension $\leqslant \operatorname{dim}_{H}\left(\operatorname{supp} \mu^{\infty}\right) \leqslant 1$.

\section{Sub-class of Image Measures}

Let $\mathcal{M}([0,1])$ denote the sub-class of probability Borel measures defined on the segment $[0,1]$ as follows (for more detials see $[1,2]$ ).

Let $Q \equiv\left\{\mathbf{q}_{k}\right\}_{k=1}^{\infty}$ be a sequence of stochastic vectors in $\mathbf{R}^{2}$ with strictly positive coordinates, $\mathbf{q}_{k}=\left(q_{0 k}, q_{1 k}\right), q_{0 k}, q_{1 k}>0, q_{0 k}+q_{1 k}=1$. We will refer to $Q$ as the infinite stochastic matrix

$$
Q=\left\{\mathbf{q}_{k}\right\}_{k=1}^{\infty}=\left(\begin{array}{lllll}
q_{01} & q_{02} & \ldots & q_{0 k} & \ldots \\
q_{11} & q_{12} & \ldots & q_{1 k} & \ldots
\end{array}\right)
$$

Given $Q$ we consider a family of closed intervals

$$
\Delta_{i_{1}}, \Delta_{i_{1} i_{2}}, \ldots, \Delta_{i_{1} i_{2} \ldots i_{k}}, \ldots \subset[0,1],\left(i_{1}, i_{2}, \ldots, i_{k}, \ldots \text { are equal to } 0 \text { or } 1\right)
$$

with lengths

$$
\left|\Delta_{i_{1}}\right|=q_{i_{1} 1},\left|\Delta_{i_{1} i_{2}}\right|=q_{i_{1} 1} \cdot q_{i_{2} 2},\left|\Delta_{i_{1} i_{2} \ldots i_{k}}\right|=q_{i_{1} 1} \cdot q_{i_{2}} \ldots q_{i_{k} k}, k \geqslant 1,
$$

and such that

$$
\begin{aligned}
{[0,1] } & =\Delta_{0} \bigcup \Delta_{1}, \\
\Delta_{i_{1}} & =\Delta_{i_{1} 0} \bigcup \Delta_{i_{1} 1},
\end{aligned}
$$

and so on for any $k$,

$$
\Delta_{i_{1} i_{2} \ldots i_{k}}=\Delta_{i_{1} i_{2} \ldots i_{k} 0} \bigcup \Delta_{i_{1} i_{2} \ldots i_{k} 1} .
$$


Assume

$$
\prod_{k=1}^{\infty} \max _{i}\left\{q_{i k}\right\}=0 .
$$

Then any $x \in[0 ; 1]$ can be represented in the following form

$$
x=\bigcap_{k=1}^{\infty} \Delta_{i_{1}(x) \cdots i_{k}(x)}=: \Delta_{i_{1}(x) \cdots i_{k}(x) \cdots} .
$$

Moreover, (2) implies that the Borel $\sigma$-algebra $\mathcal{B}$ on $[0 ; 1]$ coincides with the $\sigma$-algebra generated by the family of subsets $\left\{\Delta_{i_{1} \cdots i_{k}}\right\}_{k=1}^{\infty}$.

To a fixed $Q$, we associate a sub-class of measures $\mathcal{M}([0 ; 1])$ with a family of all sequences of stochastic vectors

$$
P=\left\{p_{k}\right\}_{k=1}^{\infty}=\left(\begin{array}{lllll}
p_{01} & p_{02} & \ldots & p_{0 k} & \ldots \\
p_{11} & p_{12} & \ldots & p_{1 k} & \ldots
\end{array}\right),
$$

where $p_{0 k}, p_{1 k} \geqslant 0, p_{0 k}+p_{1 k}=1$. Namely, we associate to each such matrix $P$ a Borel measure $\mu \in \mathcal{M}([0 ; 1])$ defined as follows.

We consider a sequence of probability spaces $\left(\Omega_{k}, \mathcal{A}_{k}, \mu_{k}^{*}\right)$, where $\Omega_{k}=\{0 ; 1\}$, $\mathcal{A}_{k}=2^{\Omega_{k}}, \mu_{k}^{*}(i)=p_{i k}$. Let $\left(\Omega, \mathcal{A}, \mu^{*}\right)$ be the infinite product of the above probability spaces. We define a measurable mapping $f$ from $\Omega$ into $[0 ; 1]$ in the following way: for any $\omega=\left(\omega_{1}, \omega_{2}, \ldots, \omega_{k}, \ldots\right) \in \Omega$ we set

$$
f(\omega)=\Delta_{\omega_{1} \omega_{2} \cdots \omega_{k} \cdots}=\bigcap_{k=1}^{\infty} \Delta_{\omega_{1} \ldots \omega_{k}},
$$

and, finally, we define the measure $\mu$ as the image measure of $\mu^{*}$ under $f$, i.e.,: for any Borel subset $E$ we put $\mu(E)=\mu^{*}\left(f^{-1}(E)\right)$, Where $f^{-1}(E)=\{\omega: f(\omega) \in E\}$.

The following results (see Theorem 1 below) on image measures are well known (see e.g. $[2,4,5]$ ). In order to formulate them we need some notations. We write, $\mu \in \mathcal{M}_{\mathrm{pp}}, \mathcal{M}_{\mathrm{ac}}, \mathcal{M}_{\mathrm{sc}}$ if the measure $\mu$ is pure point, pure absolutely continuous, or pure singular continuous, resp. Further, for the above $Q$ and $P$ we define

$$
P_{\max }(\mu):=\prod_{k=1}^{\infty} \max _{i}\left\{p_{i k}\right\}
$$

and

$$
\rho(\mu, \lambda):=\prod_{k=1}^{\infty}\left(\sqrt{p_{0 k} \cdot q_{0 k}}+\sqrt{p_{1 k} \cdot q_{1 k}}\right) .
$$

Theorem 1. Each measure $\mu \equiv \mu_{p} \in \mathcal{M}([0,1])$ is of pure type:

(a) $\mu \in \mathcal{M}_{\mathrm{pp}}$ iff $P_{\max }(\mu)>0$,

(b) $\mu \in \mathcal{M}_{\text {ac }}$ iff $\rho(\mu, \lambda)>0$,

(c) $\mu \in \mathcal{M}_{\mathrm{sc}}$ iff $P_{\max }(\mu)=0$ and $\rho(\mu, \lambda)=0$. 
In the continuous case the measure $\mu$ can also be defined in the following simple way. We define a measure $\hat{\mu}$ on the semi-ring of subsets of the form $\hat{\Delta}_{i_{1} \ldots i_{k}}=[a ; b)$, where, $a=\Delta_{i_{1} \ldots i_{k}(0)} ; b=\Delta_{i_{1} \ldots i_{k}(1)}$ : we put

$$
\hat{\mu}\left(\hat{\Delta}_{i_{1} \ldots i_{k}}\right)=p_{i_{1} 1} \cdots p_{i_{k} k}
$$

The extension $\tilde{\mu}$ of $\hat{\mu}$ on any Borel subset of $[0 ; 1)$ is defined in the usual way. We put, finally $\bar{\mu}(E)=\tilde{\mu}(E \cap[0 ; 1))$ for any Borel subset of $[0 ; 1]$. It is not hard to prove that $\bar{\mu} \equiv \mu$.

\section{Conflict Interaction Between Image Measures}

We define the non-commutative conflict composition * with two positions for a couple of stochastic vectors $\mathbf{p}, \mathbf{r} \in \mathbf{R}^{2}$ as follows:

$$
\mathbf{p}^{1}:=\mathbf{p} * \mathbf{r}, \quad \mathbf{r}^{1}:=\mathbf{r} * \mathbf{p},
$$

where the coordinates of the vectors $\mathbf{p}^{1}, \mathbf{r}^{1}$ are given by the formulae:

$$
p_{i}^{(1)}:=\frac{p_{i}\left(1-r_{i}\right)}{1-(\mathbf{p}, \mathbf{r})}, \quad r_{i}^{(1)}:=\frac{r_{i}\left(1-p_{i}\right)}{1-(\mathbf{p}, \mathbf{r})}, i=0,1,
$$

where $(\mathbf{p}, \mathbf{r})$ stands for the inner product in $\mathbf{R}^{2}$. Obviously we have to exclude the case $(\mathbf{p}, \mathbf{r})=1$.

The iteration of the composition * generates a dynamical system in the space $\mathbf{R}^{2} \times \mathbf{R}^{2}$ defined by the mapping:

$$
g:\left(\begin{array}{c}
\mathbf{p}^{N-1} \\
\mathbf{r}^{N-1}
\end{array}\right) \rightarrow\left(\begin{array}{l}
\mathbf{p}^{N} \\
\mathbf{r}^{N}
\end{array}\right), N \geq 1, \mathbf{p}^{0} \equiv \mathbf{p}, \mathbf{r}^{0} \equiv \mathbf{p},
$$

where the coordinates of $\mathbf{p}^{N}, \mathbf{r}^{N}$ are defined by induction,

$$
p_{i}^{(N)}:=\frac{p_{i}^{N-1}\left(1-r_{i}^{(N-1)}\right)}{z^{N-1}}, \quad r_{i}^{(N)}:=\frac{r_{i}^{N-1}\left(1-p_{i}^{(N-1)}\right)}{z^{N-1}}, i=0,1,
$$

with $z^{N-1}=1-\left(\mathbf{p}^{N-1}, \mathbf{r}^{N-1}\right)>0$.

Lemma. ([6, 7]). For each pair of stochastic vectors $p, r \in(R)^{2},(p, r) \neq 1$, the following limits exist and are invariant with respect to *:

$$
\mathbf{p}^{\infty}=\lim _{N \rightarrow \infty} \mathbf{p}^{N}, \mathbf{r}^{\infty}=\lim _{N \rightarrow \infty} \mathbf{r}^{N}
$$

Moreover one has:

$$
\begin{array}{ll}
\mathbf{p}^{\infty}=(1,0), \mathbf{r}^{\infty}=(0,1) & \text { iff } p_{0}>r_{0}, \\
\mathbf{p}^{\infty}=(0.1), \mathbf{r}^{\infty}=(1,0) & \text { iff } p_{1}>r_{1}, \\
\mathbf{p}^{\infty}=\mathbf{r}^{\infty}=(1 / 2,1 / 2), & \text { iff } p_{0}=r_{0}, p_{1}=r_{1} .
\end{array}
$$


We will introduce now a non-commutative conflict interaction (in the sense of $[6,7])$ between image measures from the sub-class $\mathcal{M}([0,1])$ and use the just presented facts for these analysis of the spectral transformations of these measures.

Let $\mu$ and $\nu$ be a couple of image measures corresponding to a pair of sequences of stochastic vectors $P^{0}=\left\{\mathbf{p}_{k}^{0}\right\}_{k=1}^{\infty}$ and $R^{0}=\left\{\mathbf{r}_{k}^{0}\right\}_{k=1}^{\infty}$, resp., i.e., $\mu=\mu_{P^{0}}, \nu=\nu_{R^{0}}$. The conflict interaction between $\mu$ and $\nu$, denoted by $*$, is by definition given by the couple $\mu^{1}, \nu^{1}$

$$
\mu^{1}:=\mu * \nu, \nu^{1}:=\nu * \mu,
$$

where $*$ is defined by using the above defined conflict compositions for stochastic vectors in $\mathbf{R}^{2}$. Namely, we associate a new couple of measures $\mu^{1}, \nu^{1} \in \mathcal{M}([0,1])$ with sequences $P^{1}=\left\{\mathbf{p}_{k}^{1}\right\}_{k=1}^{\infty}$ and $R^{1}=\left\{\mathbf{r}_{k}^{1}\right\}_{k=1}^{\infty}$, where the coordinates of vectors $\mathbf{p}_{k}^{1}, \mathbf{r}_{k}^{1}$ are defined according to formulae (3), i.e.,

$$
p_{i k}^{(1)}:=\frac{p_{i k}\left(1-r_{i k}\right)}{1-\left(\mathbf{p}_{k}, \mathbf{r}_{k}\right)}, \quad r_{i k}^{(1)}:=\frac{r_{i k}\left(1-p_{i k}\right)}{1-\left(\mathbf{p}_{k}, \mathbf{r}_{k}\right)}, \quad i=0,1, k=1,2, \ldots
$$

where $p_{i k} \equiv p_{i k}^{(0)}, r_{i k} \equiv r_{i k}^{(0)}$ and $\mathbf{r}_{k} \equiv \mathbf{r}_{k}^{0}, \mathbf{r}_{k} \equiv \mathbf{r}_{k}^{0}$. Of course we assume that

$$
\left(\mathbf{p}_{k}^{0}, \mathbf{r}_{k}^{0}\right) \neq 1, \quad k=1,2, \ldots
$$

By induction we introduce the sequences $P^{N}=\left\{\mathbf{p}_{k}^{1}\right\}_{k=1}^{\infty}$ and $R^{N}=\left\{\mathbf{r}_{k}^{N}\right\}_{k=1}^{\infty}$ for any $\mathrm{N}=1,2, \ldots$, where the stochastic vectors $\mathbf{p}_{k}^{N}=\mathbf{p}_{k}^{N-1} * \mathbf{r}_{k}^{N-1}, \mathbf{r}_{k}^{N}=\mathbf{r}_{k}^{N-1} * \mathbf{p}_{k}^{N-1}$ are defined as $N$-times iterations of the composition $\%$; the coordinates of the vectors $\mathbf{p}_{k}^{N}, \mathbf{r}_{k}^{N}$ are calculated by formulae like (5).

Further, with each pair $P^{N}, R^{N}$ we associate a couple of image measures $\mu^{N} \equiv \mu_{P^{N}}$ and $\nu^{N} \equiv \nu_{R^{N}}$ from the class $\mathcal{M}([0,1])$. Therefore the mapping $g$ generates the dynamical system in the space $\mathcal{M}([0,1]) \times \mathcal{M}([0,1])$ :

$$
U(g):\left(\begin{array}{l}
\mu^{N-1} \\
\nu^{N-1}
\end{array}\right) \rightarrow\left(\begin{array}{l}
\mu^{N} \\
\nu^{N}
\end{array}\right)
$$

We are interesting in the existence and structure of the invariant points of the so defined dynamical system.

Theorem 2. For each couple of image measures $\mu \equiv \mu_{P^{0}}, \nu \equiv \nu_{R^{0}}$, under condition (7), there exist two limiting invariant measures,

$$
\mu^{\infty}=\lim _{N \rightarrow \infty} \mu^{N}, \nu^{\infty}=\lim _{N \rightarrow \infty} \nu^{N} .
$$

The measures $\mu^{\infty}, \nu^{\infty}$ are mutually singular iff $P^{0} \neq R^{0}$, and $\mu^{\infty}, \nu^{\infty}$ are identical iff $P^{0}=R^{0}$.

Proof. This follows easily from Lemma.

Our goal here is to investigate the properties of the limiting measures. 


\section{Metric Properties}

Let us introduce two sets for a couple of probability image measures $\mu, \nu . N_{=}:=$ $\left\{k: \mathbf{p}_{\mathbf{k}}=\mathbf{r}_{\mathbf{k}}\right\}$, and $\mathbf{N}_{\neq}:=\mathbf{N} / \mathbf{N}_{=} \equiv\left\{k: \mathbf{p}_{k} \neq \mathbf{r}_{k}\right\}$, and put

$$
\begin{aligned}
Q_{=} & :=\sum_{k \in N_{=}}\left[\left(1-2 q_{0 k}\right)^{2}+\left(1-2 q_{1 k}\right)^{2}\right], \\
W_{\neq}(\mu) & :=\sum_{k \in N_{\neq}} q_{(i) k}, \quad \text { where } q_{(i) k}=\left\{\begin{array}{l}
q_{0 k}, \text { if } p_{0 k}<r_{0 k} \\
q_{1 k}, \text { if } p_{1 k}<r_{1 k}
\end{array},\right. \\
W_{\neq}(\nu) & :=\sum_{k \in N_{\neq}}\left(1-q_{(i) k}\right) .
\end{aligned}
$$

Theorem 3. (a) $\mu^{\infty} \in \mathcal{M}_{p p}$, iff $\left|\mathbf{N}_{=}\right|<\infty$.

(b) $\mu^{\infty} \in \mathcal{M}_{a c}$, iff $Q_{=}<\infty$ and $W_{\neq}<\infty$.

(c) $\mu^{\infty} \in \mathcal{M}_{s c}$, iff $\left|\mathbf{N}_{=}\right|=\infty$ and at least one of the conditions, $W_{\neq}(\mu)=\infty$, or $Q==\infty$, is fulfilled.

Proof. (a) By Theorem 1 the measure $\mu^{\infty}$ belongs to $\mathcal{M}_{\mathrm{pp}}$ iff

$$
P_{\max }\left(\mu^{\infty}\right):=\prod_{k \in N} \max _{i}\left\{p_{i k}^{\infty}\right\}>0
$$

Since for each vector $\mathbf{p}_{k}^{\infty} \in \mathbf{R}^{2}$ the coordinates $p_{i k}^{(\infty)}$ are equal to $0,1 / 2$, or 1 , we have

$$
\max _{i} p_{i k}^{(\infty)}= \begin{cases}1 / 2, & \text { if } k \in \mathbf{N}_{=} \\ 1, & \text { if } k \in \mathbf{N}_{\neq}\end{cases}
$$

Hence $\mu^{\infty} \in \mathcal{M}_{\mathrm{pp}}$ if and only if $\mathbf{p}_{k} \neq \mathbf{r}_{k} \forall k>k_{0}$ fo some $k_{0} \in N$. This means that supp $\mu$ consists of at most $2^{\left|N_{=}\right|}$points.

(b) Let $\left|\mathbf{N}_{=}\right|=\infty$ and, therefore, $\mu^{\infty} \in \mathcal{M}_{\mathrm{ac}} \cup \mathcal{M}_{\mathrm{sc}}$. By Theorem 1, $\mu^{\infty} \in \mathcal{M}_{\mathrm{ac}}$ iff

$$
\rho\left(\mu^{\infty}, \lambda\right)=\prod_{k=1}^{\infty}\left(\sqrt{p_{0 k}^{(\infty)} q_{0 k}}+\sqrt{p_{1 k}^{(\infty)} q_{1 k}}\right)>0 .
$$

Taking into account that $p_{0 k}^{(\infty)}=p_{1 k}^{(\infty)}=\frac{1}{2}$ for all $k \in \mathbf{N}_{=}$, and $p_{0 k}^{(\infty)}=1$ iff $p_{0 k}>r_{0 k}, p_{0 k}^{(\infty)}=0$, iff $p_{0 k}<r_{0 k}$, we have

$$
\sqrt{p_{0 k}^{(\infty)} q_{0 k}}+\sqrt{p_{0 k}^{(\infty)} q_{1 k}}= \begin{cases}\sqrt{\frac{1}{2} q_{0 k}}+\sqrt{\frac{1}{2} q_{1 k}}, & \text { iff } k \in \mathbf{N}_{=}, \\ \sqrt{1-q_{(i) k}}, & \text { iff } k \in \mathbf{N}_{\neq} .\end{cases}
$$


Therefore, $\mu^{\infty} \in \mathcal{M}_{\text {ac }}$ iff

$$
\begin{aligned}
\prod_{k \in \mathbf{N}_{=}} & \left(\sqrt{\frac{1}{2} q_{0 k}}+\sqrt{\frac{1}{2} q_{1 k}}\right) \cdot \prod_{k \in \mathbf{N}_{\neq}}\left(\sqrt{1-q_{(i) k}}\right)>0 \\
& \Leftrightarrow\left\{\begin{array}{l}
\prod_{k \in \mathbf{N}_{=}}\left(\sqrt{\frac{1}{2} q_{0 k}}+\sqrt{\frac{1}{2} q_{1 k}}\right)>0, \\
\prod_{k \in \mathbf{N}_{\neq}}\left(\sqrt{1-q_{(i) k}}\right)>0 .
\end{array}\right.
\end{aligned}
$$

By using simple arguments, we have

$$
\prod_{k \in \mathbf{N}_{\neq}}\left(\sqrt{1-q_{(i) k}}\right)>0 \Leftrightarrow \prod_{k \in \mathbf{N}_{\neq}}\left(1-q_{(i) k}\right)>0 \Leftrightarrow \sum_{k \in \mathbf{N}_{\neq}} q_{(i) k}<\infty,
$$

and

$$
\begin{aligned}
& \prod_{k \in \mathbf{N}_{=}}\left(\sqrt{\frac{1}{2} q_{0 k}}+\sqrt{\frac{1}{2} q_{1 k}}\right)>0 \Leftrightarrow \prod_{k \in \mathbf{N}_{=}}\left(\frac{1}{2}+\sqrt{q_{0 k} q_{1 k}}\right)>0 \\
& \Leftrightarrow \sum_{k \in \mathbf{N}_{=}}\left(\frac{1}{2}-\sqrt{q_{0 k} q_{1 k}}\right)<\infty \Leftrightarrow \sum_{k \in \mathbf{N}}\left(1-2 \sqrt{q_{0 k} q_{1 k}}\right)<\infty \\
& \Leftrightarrow \prod_{k \in \mathbf{N}_{=}} 4 q_{0 k} q_{1 k}>0 \Leftrightarrow \prod_{k \in \mathbf{N}_{=}}\left(1-\left(1-2 q_{0 k}\right)^{2}\right)>0 \\
& \Leftrightarrow \sum_{k \in \mathbf{N}_{=}}\left(1-2 q_{0 k}\right)^{2}<\infty \Leftrightarrow \sum_{k \in \mathbf{N}_{=}}\left(1-2 q_{1 k}\right)^{2}<\infty
\end{aligned}
$$

Therefore,

$$
\mu^{\infty} \in \mathcal{M}_{a c} \Leftrightarrow\left\{\begin{array}{l}
W_{\neq}(\mu)<\infty, \\
Q_{=}<\infty .
\end{array}\right.
$$

(c) If $\left|\mathbf{N}_{=}\right|=\infty$, then from (a) the continunity of $\mu^{\infty}$ follows easily. If $Q_{=}(\mu)=\infty$ or $W_{\neq}(\mu)=\infty$, then from (b) it follows that $\mu \perp \lambda$, and therefore $\mu^{\infty} \in \mathcal{M}_{s c}$. Conversely, if $\mu^{\infty} \in \mathcal{M}_{a c}$, then $\mu^{\infty}$ is a continuous measure and $\mu^{\infty} \perp \lambda$.

The continunity of $\mu^{\infty}$ implies $\left|\mathbf{N}_{=}\right|=\infty$. Since $\mu^{\infty} \perp \lambda$, we have $\rho\left(\mu^{\infty}, \lambda\right)=$ 0 and, therefore $Q_{=}=\infty$ or $W_{\neq}(\mu)=\infty$.

Remarks. (1) The Theorem holds for the measure $\nu^{\infty}$ if $W_{\neq}(\mu)$ is replaced by $W_{\neq}(v)$.

(2) Both measure $\mu^{\infty}, \nu^{\infty}$ belong to $\mathcal{M}_{a c}$ only in the case $\left|\mathbf{N}_{\neq}\right|<\infty$ provided that $Q=<\infty$, because, if $\left|\mathbf{N}_{\neq}\right|=\infty$ then at least one of values $W_{\neq}(\mu)$ or $W_{\neq}(\nu)$ is infinite.

(3) The condition $Q_{=}<\infty$ is fulfilled iff

$$
\sum_{k \in \mathbf{N}=}\left(1-2 q_{0 k}\right)^{2}<\infty
$$


Example 1. Let $q_{0} k=q_{1} k=1 / 2$ for all $k \in N$. Then $Q_{=}=0$ for any $\mathbf{N}_{=}$and $W_{\neq}(\mu)<\infty$ iff $\left|\mathbf{N}_{\neq}\right|=\infty$. Thus both measures $\mu^{\infty}, v^{\infty}$ belong to $\mathcal{M}_{p} p$ iff $\left|\mathbf{N}_{=}\right|=\infty$. Moreover $\mu^{\infty}, v^{\infty} \in \mathcal{M}_{a c}$ iff $\left|\mathbf{N}_{\neq}\right|=\infty$, and $\mu^{\infty}, v^{\infty} \in \mathcal{M}_{s c}$ iff $\left|\mathbf{N}_{\neq}\right|=\infty$ and $\left|\mathbf{N}_{=}\right|=\infty$.

Example 2. Let $q_{0 k} \notin\left(\frac{1}{2}-\varepsilon ; \frac{1}{2}+\varepsilon\right)$ for some $\varepsilon>0$. Then $Q=<\infty$ only if $\left|\mathbf{N}_{=}\right|=\infty$. Thus $\mu^{\infty}, v^{\infty} \in \mathcal{M}_{p p}$ iff, $\left|\mathbf{N}_{=}\right|<\infty$, and $\mu^{\infty}, v^{\infty} \in \mathcal{M}_{s c}$ iff $\left|N_{=}\right|=\infty$, but one never has $\mu^{\infty}, \nu^{\infty} \in \mathcal{M}_{a c}$.

\section{Topological Properties}

A Borel measure $\mu$ on $\mathbf{R}$ is of the $\mathrm{S}$-type if its support, supp $\mu \equiv S_{\mu}$, is a regularly closed set, i.e.,

$$
S_{\mu}=\left(\operatorname{int} S_{\mu}\right)^{c l},
$$

where int $A$ denotes the interior part of the set $\mathrm{A}$, and $(E)^{c l}$ denotes the closure of the set $E$. A measure $\mu$ is of the C-type if its support $S_{\mu}$ is aset of zero Lebesgue measure. A measure $\mu$ is of the $P$-type if its support $S_{\mu}$ is a nowhere dense set and $S_{\mu}$ has a positive Lebesgue measure in any small neighbourhood of each point $x$ from $S_{\mu}$, i.e.,

$$
\forall x \in S_{\mu}, \forall_{\varepsilon}>0: \lambda\left(B(x, \varepsilon) \bigcap S_{\mu}\right)>0
$$

we shall write $(\operatorname{cf} . w i t h[1,9]) \mu \in \mathcal{M}^{S}$, resp. $\mathcal{M}^{C}$, or resp. $\mathcal{M}^{P}$ if $\mu$ is of the $S-$, resp. $C$-or resp. $P$-type.

Theorem 4. The infinite conflict interaction between two image measures $\mu, v \in$ $([0,1])$ produces limiting measures $\mu^{\infty}, v^{\infty}$ of pure topological type. We have:

(a) $\mu^{\infty} \in \mathcal{M}^{S}$, iff $\left|\mathbf{N}_{\neq}\right|<\infty$,

(b) $\mu^{\infty} \in \mathcal{M}^{C}$, iff $W_{\neq}(\mu)=\infty$,

(c) $\mu^{\infty} \in \mathcal{M}^{P}$, iff $\left|\mathbf{N}_{\neq}\right|=\infty$ and $W_{\neq}(\mu)<\infty$.

Proof. (a) By Theorem 8 in[2] the measure $\mu^{\infty}$ is of the S-type iff the matrix $P^{\infty}$ contains only a finite number of zero elements. This is possible iff $\left|\mathbf{N}_{\neq}\right|<\infty$.

(b) The measure $\mu^{\infty}$ is of the C-type (see Theorem 8 in [2]) iff the matrix $P^{\infty}$ contains infinitely many columns having elements $p_{i k}=0$, and besides, $\sum_{k=1}^{\infty}\left(\sum_{i: p i k=0} q_{i k}\right)=\infty$, that is equivalent to $\left|\mathbf{N}_{\neq}\right|=\infty$ and $W_{\neq}(\mu)=\infty$. Since $\prod_{k=1}^{\infty} \operatorname{maxq}_{i k}=0$, we conclude that from $W_{\neq}=\infty$ it follows that $\left|\mathbf{N}_{\neq}\right|=\infty$.

(c) Finally the measure $\mu^{\infty}$ is of the $P$-type (see again Theorem 8 in [2]) iff the matrix $P \infty$ contains infinitely many columns with zero elements $p_{i k}$, and moreover, $\sum_{k=1}^{\infty}\left(\sum_{i: p i k=0} q_{i k}\right)<\infty$, i.e., $\left|\mathbf{N}_{\neq}\right|=\infty$ and $W_{\neq}(\mu)<\infty$. 
Remarks. (1) The assertions of Theorems 4 are also true for the measure $v^{\infty}$ if one replaces $W_{\neq}(\mu)$ by $W_{\neq}(v)$.

(2) It is not possible for the measures $\mu^{\infty}, v^{\infty}$ to be both of the $P$-type. So if one of them is of the $P$-type, then the other is necessarily of the $C$-type.

The combinations of Theorems 3 and 4 leads to

\section{Corollaries.}

(a) The set $M_{p p} \bigcap \mathcal{M}^{s}$ is empty.

$\mu^{\infty} \in \mathcal{M}_{p p} \cap \mathcal{M}^{c} \quad$ iff $\left|\mathbf{N}_{=}\right|<\infty$.

The set $\mathcal{M}_{p p} \cap \mathcal{M}^{P}$ is empty.

(b) $\mu^{\infty} \in \mathcal{M}_{a c} \bigcap \mathcal{M}^{s}$ iff $\left|\mathbf{N}_{=}\right|<\infty$, and $Q_{=}<\infty$.

The set $\mathcal{M}_{a c} \cap \mathcal{M}^{c}$ is empty.

$\mu^{\infty} \in \mathcal{M}_{a c} \bigcap \mathcal{M}^{P}$ iff $\left|\mathbf{N}_{=}\right|=\infty,\left|\mathbf{N}_{\neq}\right|=\infty$, but $Q_{=}<\infty$ and $W_{\neq}(\mu)<\infty$.

(c) $\mu^{\infty} \in \mathcal{M}_{s c} \cap \mathcal{M}^{S}$ iff $\left|\mathbf{N}_{\neq}\right|<\infty$, and $Q==\infty$.

$\mu^{\infty} \in \mathcal{M}_{s c} \bigcap \mathcal{M}^{C} \quad$ iff $W_{\neq}(\mu)=\infty$.

$\mu^{\infty} \in \mathcal{M}_{s c} \cap \mathcal{M}^{P} \quad$ iff $\left|\mathbf{N}_{\neq}\right|=\infty, W_{\neq}(\mu)<\infty$ and $Q_{=}=\infty$.

Proof. (a) $\mathcal{M}_{p p} \bigcap \mathcal{M}^{S}=\emptyset$ since $\left|\mathbf{N}_{\neq}\right|<\infty$ and $\left|\mathbf{N}_{=}\right|<\infty$ are mutually exclusive conditions. So, if $\left|\mathbf{N}_{=\mid}\right|<\infty$, then $\left|\mathbf{N}_{\neq}\right|<\infty$ and $\mu^{\infty} \in \mathcal{M}^{C} . \mathcal{M}_{p p} \cap \mathcal{M}^{P}=\emptyset$, since $W_{\neq}(\mu)<\infty$ with $\left|\mathbf{N}_{=}\right|<\infty$ mean that $\prod_{k=1}^{\infty} q_{i k}>0$, but this contradicts our assumption (2).

(b) The first assertion is evident since $\left|\mathbf{N}_{\neq}\right|<\infty$ implies $W_{\neq}(\mu)<\infty$. Further $\mathcal{M}_{a c} \bigcap \mathcal{M}^{C}=\emptyset$ since the conditions $W_{\neq}(\mu)<\infty$ and $W_{\neq}(\mu)=\infty$ can not be simultaneously fulfilled. Finally, in the case $\left|\mathbf{N}_{\neq}\right|=\infty, \mu^{\infty} \in \mathcal{M}_{a c}$ iff $\mu^{\infty} \in \mathcal{M}^{P}$

(c) In spite of that $W_{\neq}(\mu)<\infty$ if $\left|\mathbf{N}_{\neq}\right|<\infty$, it is still possible that $Q_{=}(\mu)=\infty$. Thus, in general, we have $\mathcal{M}_{\mathrm{sc}} \bigcap \mathcal{M}^{S} \neq \emptyset$.

Moreover, if $\left|\mathbf{N}_{\neq}\right|=\infty$, then $W_{\neq}(\mu)=\infty$ implies $\mu^{\infty} \in \mathcal{M}_{\mathrm{sc}} \cap \mathcal{M}^{C}$.

But if $\left|\mathbf{N}_{\neq}\right|<\infty$ and $W_{\neq}(\mu)=\infty$, then it still possible that $Q_{=}(\mu)=\infty$, or equivalently, $\mu^{\infty} \in \mathcal{M}_{\mathrm{sc}} \cap \mathcal{M}^{P}$.

Remarks. Of course, all corollaries are true for the measure $\nu^{\infty}$ if one replaces $W_{\neq}(\mu)$ by $W_{\neq}(\nu)$.

The measures $\mu^{\infty}, \nu^{\infty}$ in general have a rather complicated local structure and their supports might posses arbitrary Hausdorff dimensions.

Let us denote by $\operatorname{dim}_{\mathrm{H}}(E)$ the Hausdorff dimension of a set $E \subset \mathbf{R}$.

Suppose $q_{0 k}=q_{1 k}=1 / 2$. 
Theorem 5. Given a number $c_{0} \in[0,1]$ let $\mu \in \mathcal{M}([0,1])$ be any probability image measure. Then there exists another probability image measusre $\nu$ such that

$$
\operatorname{dim}_{\mathrm{H}}\left(\operatorname{supp} \mu^{\infty}\right)=c_{0} .
$$

Proof. Let $\left|\mathbf{N}_{\neq, k}\right|=\left|\mathbf{N}_{\neq} \bigcap\{1,2, \ldots, k\}\right|$ denote the cardinality of the set $\mathbf{N}_{\neq, k}:=$ $\left\{s \in \mathbf{N}_{\neq}: s \leqslant k\right\}$. Clearly $\left|\mathbf{N}_{\neq, k}\right|+\left|\mathbf{N}_{=, k}\right|=k$, where $\mathbf{N}_{=, k}:=\left\{s \in \mathbf{N}_{=}: s \leqslant k\right\}$.

By Theroem 2[8] the Hausdorff dimension of the set supp $\mu^{\infty}$ may be calculated by the formula:

$$
\operatorname{dim}_{\mathrm{H}}\left(\operatorname{supp} \mu^{\infty}\right)=\liminf _{k \rightarrow \infty} \frac{\left|\mathbf{N}_{=, k}\right|}{k} .
$$

Given the stochastic matrix $P$ corresponding to the starting measure $\mu$ one always can chose (in a non-unique way) another stochastic matrix $R$ (uniquely associated with a measure $\nu$ ) such that the condition $\lim _{k \rightarrow \infty} \frac{\left|\mathbf{N}_{=, k}\right|}{k}=c_{0}$ will be satisfied.

\section{Acknowledegments}

This work was partly supported by DFG 436 UKR 113/53, DFG 436 UKR 113/67, INTAS 00-257, SFB-611 projects and by UFFI 01.07/00081 grant.

\section{References}

[1] S. Albeverio, V. Koshmanenko, G. Torbin, Fine structure of the singular continuous sperctrum, Meth. Funct. Anal. Topol. 9(2) (2003), 101-127.

[2] S. Albeverio, V. Koshmanenko, M. Pratsiovytyi, and G. Tprbin, Q̃-representation of real numbers and fractal probability distribution, subm. to Infinite Dimensiional Analysis, Quantum Probability and Related Topics.

[3] M. F. Barnsley, and S. Demko, Iterated functional system and the global constuction of fractals, Proc. Roy. Soc. London A, 399 (1985), 243-275.

[4] S. D. Chatterji, Certain induced measures on the unit interval, J. London Math. Soc., 38 (1962), 325-331.

[5] S. Kakutani, Equivalence of infinite product measures, Ann. Math., 49 (1948), $214-224$.

[6] V. Koshmanenko, The theorem of conflicts for a pair of stochastic vectors, Ukrainian Math. J., 55(4) (2003), 555-560.

[7] V. Koshmanenko, The theorem of conflicts for a couple of probability measures, accepted to publ. in Math. Meth. Oper. Res., 59(2) (2004).

[8] M. V. Pratsiovytyi, Fractal, superfractal and anomalously fractal distribution of random variables with a fixed infinite set of independent $n$-adic digits, Exploring Stochastic Laws VSP (1995), 409-416.

[9] M. V. Pratsiovytyi, Fractal approach to investigation of singular distributions (in Ukrainian) National Pedagogical Univ., Kyiv, 1998. 
Sergio Albeverio

Institut für Angewandte Mathematik

Universität Bonn

Bonn, Germany

and

BiBos

Bielefeld-Bonn, Universität Bielefeld

Bielefeld, Germany

and

SFB-611

Universität Bonn

Bonn, Germany

and

IZKS

Bonn, Germany

and

CERFIM

Locarno

and Acc. Arch. Locarno

and USI

Switzerland

E-mail: albeverio@uni-bonn.de

Volodymyr Koshmonenko

Institute of Mathematics

Kyiv, Ukraine

E-mail: kosh@imath.kiev.net

Mykola Pratsiovytyi, Grygoriy Torbin

National Pedagogical University

Kyiv, Ukraine

E-mail: prats@ukrpost.net, torbin@imath.kiev.ua

Received 27 August 2003; accepted 15 December 2003

To access this journal online:

(ID) http://www.birkhauser.ch 